VLSI Design

1995, Vol. 3, No. 2, pp. 115-129

Reprints available directly from the publisher

Photocopying permitted by license only (c) 1995 OPA (Overseas Publishers Association)

Amsterdam B.V. Published under license by

Gordon and Breach Science Publishers SA

\title{
On the Structure and Closure-Condition of the Hydrodynamic Model
}

\author{
MASSIMO RUDAN and GIORGIO BACCARANI \\ Dipartimento di Elettronica, Informatica e Sistemistica, Università di Bologna \\ Viale Risorgimento 2, 40136 Bologna, Italy
}

(Received October 3, 1994)

\begin{abstract}
Basing on a general formulation of the hydrodynamic model for semiconductor devices [1], a consistent approach to the solution of the model equations is proposed, along with a method for selecting the closure condition. The definition of the coefficients of the hydrodynamic and energy-transport models is then re-examined in order to make a comparison between them possible. Finally, the closure condition is discussed and compared with others proposed in the literature. ${ }^{1}$
\end{abstract}

Key Words: Semiconductor Devices, Transport, Hydrodynamic Model, Closure Condition, Relaxation Times.

\section{INTRODUCTION}

$\mathbf{T}$ The hydrodynamic (HD) model has become a useful tool for the simulation of semiconductor devices. Based for instance on the discretization scheme depicted in [2], one- and two-dimensional implementations have become available $[3,4]$, which in turn have been exploited to further investigate some physical aspects [5, 6]. More recently, the implementation has been extended to three dimensions and the physical model incorporates the lattice temperature as well [7]. Comparisons with Monte Carlo analysis and experimental results (see, e.g., $[1,5,6])$ show that the distribution of the electric potential, carriers' concentration, and average energy in realistic structures can be obtained at an acceptable computational burden, thus making the model considerably helpful in the design of VLSI integrated circuits. For instance, phenomena such as velocity overshoot and impact ionization are simulated to such an extent as to provide a quantitative indication of substrate currents and breakdown voltages. The spatial distribution of the average energy

\footnotetext{
${ }^{1}$ This work was part of ADEQUAT (JESSI BT1B) and was funded as ESPRIT Project 7236.
}

of the particles made available by the model can be exploited for the estimate of carrier injection into the gate oxide (the injection typically occurs when the carrier average energy becomes considerably larger than that of the lattice, which is referred to as carrier heating). The usefulness of the hydrodynamic model makes it desirable to deepen the investigation on both the mathematical and physical aspects of it. It is also useful to compare its features with those of other models incorporating the description of the carrier average energy, such as the energy-transport (ET) model [8].

An important point of interest, related to the so-called closure condition, is inherent in the derivation of the model's equations as moments of the Boltzmann Transport Equation (BTE). The problem can qualitatively be illustrated with reference to a moment of any rank, say $M$. It is found that one of the terms of the equations contains a moment of the distribution function of higher rank than $M$, i.e., the unknown of the equation of rank $M+1$ [1]. Since each equation is then linked to the higher-rank one, the number of unknowns in the set of equations for the moments of rank 0 through $M$ always exceeds the number of equations themselves. To prevent the resulting system of PDE's from being underdetermined, it is necessary to add a further equation 
containing the first $M+1$ moments of the distribution function at most, but not the moment of rank $M+2$. This equation constitutes the closure condition, and may be derived either by simplifying the equation of rank $M+1$ or by invoking some physical reasoning independent of the derivation of the moments themselves. In the case of the hydrodynamic model the moments of rank $M=0,1$, and 2 are used, and for the closure condition it is typically assumed that the heat flux of the carriers is proportional to the gradient of the carrier temperature (Fourier law) where, in turn, the thermal conductivity depends on the carrier temperature via the Wiedmann-Franz law. It is important to point out that in general, due to the form of the moment equations, the closure condition determines the structure (or at least some coefficients) of the PDE constituting the highest-rank moment. A carelesslychosen condition may then make the solution deviate from that obtained by a direct method, such as the Monte Carlo one. One example of this is the so-called spurious velocity overshoot, whose sensitivity to the coefficient of the closure condition has been analyzed in [5]. Attempts to eliminate this phenomenon have been made, e.g., by compensation, namely by selecting a mobility model such that the overshoot is almost exactly canceled [9]. Sounder investigations based on the Scattering Matrix Approach (SMA) showed that the Wiedmann-Franz law becomes inaccurate near the carrier-temperature peaks [10] - which is where the overshoot occurs - pointing out the necessity of improving the closure condition. An attempt in this direction has recently been proposed in [11].

Sections 2 and 3 are devoted to illustrating and analyzing the structure of the moments method, to the purpose of determining a general scheme for its numerical solution and discussing the closure condition. Two examples of application are given in section 4 , with reference to the drift-diffusion model and to an extended form of the hydrodynamic model. In section 5 the energy-transport model is illustrated and compared with the hydrodynamic one. Further discussion on the closure condition is carried out in section 6 and, finally, the conclusions are drawn in section 7 .

\section{THE MOMENT EQUATIONS}

Following the derivation of [1], the expression of the moment of rank $M \geq 1$ of BTE, over the Brillouin zone and for the conduction band of a semiconduc- tor, reads

$$
\begin{array}{r}
\operatorname{div}\left(n \mathscr{R}_{M+1}\right)+n\left(\frac{q}{\hbar} \overline{\mathscr{G}}_{M}+\mathscr{V}_{M}\right) \\
=-n \mathscr{N}_{2 M} \cdot\left(\overline{\mathscr{K}}_{M}-\overline{\mathscr{K}}_{M}^{\mathrm{eq}}\right) .
\end{array}
$$

The meaning of the symbols is illustrated in the following:

$$
\mathscr{R}_{M+1}=\frac{1}{M_{C}} \sum_{r=1}^{M_{C}}\left(\overline{\mathscr{K}_{M} c_{n}}\right)_{r}, \quad \overline{\mathscr{G}}_{M}=\frac{1}{M_{C}} \sum_{r=1}^{M_{C}} \overline{\mathscr{G}}_{M r},
$$

and

$$
\mathscr{V}_{M}=\frac{1}{M_{C}} \sum_{r=1}^{M_{C}} \mathbf{v}_{n r} \cdot \operatorname{grad} \overline{\mathscr{K}}_{M r}
$$

where $M_{C}$ is the number of absolute minima of the conduction band and

$$
\mathbf{v}_{n r}=\frac{\int_{B_{r}} \mathbf{u} f_{r} \mathrm{~d}^{3} k}{\int_{B_{r}} f_{r} \mathrm{~d}^{3} k}, \quad \mathbf{c}_{n r}=\mathbf{u}-\mathbf{v}_{n r}
$$

is the average velocity of the electrons in the region $B_{r}$ (valley) around the $r$ th minimum (in turn, $\mathbf{u}$ and $\mathbf{c}_{n r}$ are the group and random velocities, and $f_{r}$ is the restriction of the distribution function $f$ to $B_{r}$ ). The bar over the symbols in (1), (2), and (3) indicates the statistical average; more precisely it is

$$
\begin{gathered}
\overline{\mathscr{K}}_{M r}=\frac{1}{n_{r}} \int_{B_{r}} \mathscr{K}_{M} f_{r} \mathrm{~d}^{3} k, \\
\left(\overline{\mathscr{K}_{M} \mathbf{c}_{n}}\right)_{r}=\frac{1}{n_{r}} \int_{B_{r}} \mathscr{K}_{M} \mathbf{c}_{n r} f_{r} \mathrm{~d}^{3} k,
\end{gathered}
$$

where $\mathscr{K}_{M}$ is a symmetrical tensor of rank $M$ made of the wave vector $\mathbf{k}$,

$$
\mathscr{K}_{0}=1, \quad \mathscr{K}_{M}=\underbrace{\mathbf{k k} \ldots \mathbf{k}}_{M \text { times }}, \quad M=1,2, \ldots
$$

and $n_{r}$ is the electron concentration in $B_{r}$. Similarly, 
it is

$$
\overline{\mathscr{G}}_{M r}(\mathbf{F})=\frac{1}{n_{r}} \int_{B_{r}} \mathscr{G}_{M} f_{r} \mathrm{~d}^{3} k,
$$

where $\mathbf{F}$ is the electric field and $\mathscr{G}$ a symmetrical tensor of rank $M$ made of the sum of $M$ terms:

$$
\begin{aligned}
\mathscr{G}_{M}(\mathbf{F})= & \text { Fkk } \ldots \mathbf{k}+\mathbf{k F k} \ldots \mathbf{k} \\
& +\cdots+\mathbf{k} \ldots \mathbf{k F k}+\mathbf{k} \ldots \mathbf{k k F} .
\end{aligned}
$$

Finally, $n=\sum_{r} n_{r}$ in (1) represents the total electron concentration, while $\mathscr{N}_{2 M}$ at the right hand side is a diagonal tensor of rank $2 M$ whose physical dimension is that of a frequency. The definition of $N_{2 M}^{\prime}$ is given by (57). Its entries are expressed by integrals involving the scattering probability and some moments of the distribution function. The inverse of $\mathscr{N}_{2 M}$ may be regarded as the macroscopic relaxation time in the moment of rank $M$. Since some of the quantities involved in its definition are unknown, $\mathscr{N}_{2 M}$ is in itself unknown. In the solution scheme, which solves the system of moments by iteration, $\mathscr{N}_{2 M}$ at the current iteration is typically approximated as a function of the moments of rank 0 through $M$, whose values are taken from the previous iteration.

In equilibrium, the term in parentheses at the right hand side of (1) vanishes. Moreover, it is $\mathbf{v}_{n r}=0$ whence $\mathscr{V}_{M}=0$. It follows

$$
\operatorname{div}\left(n \mathscr{R}_{M+1}\right)+n \frac{q}{\hbar} \overline{\mathscr{G}}_{M}=0 .
$$

Since the divergence term in the moment equations represents the diffusive effect, one sees that, from a macroscopic viewpoint, the equilibrium condition is achieved by a balance between the drift term, proportional to the carrier concentration and linearly dependent on the electric field, and the diffusive term due to the spatial non-uniformity of the concentration and/or the random velocity of the carriers. Another observation is that $\overline{\mathscr{H}}_{M}^{\text {eq }}=0$ when $M$ is odd. The hypotheses under which the above model has been derived are:

1. The restriction to $B_{r}$ of the collision term of the BTE is expressed as

$$
C_{r}=C_{r}^{\prime}-\sum_{s=1}^{M_{C}} \frac{f_{r}-\tilde{f}_{r s}}{\tau_{r s}},
$$

where $C_{r}^{\prime}$ represents the interband-transition term and $\tau_{r s}$ is the microscopic relaxation time for the transitions between the $r$ th and $s$ th valley. The terms intravalley and intervalley denote the transitions with $s=r$ and $s \neq r$, respectively. The corresponding frequencies are

$$
v_{r s}=\frac{1}{\tau_{r s}} .
$$

2. The frequencies associated to the interbandtransition terms are assumed negligible with respect to the $v_{r s}$.

3. All $M_{C}$ minima of the conduction band are equivalent to each other and the electrons are equally distributed among the valleys, whence

$$
n_{r}=\frac{1}{M_{C}} n
$$

One sees, in fact, that the averaging factor in (2), (3) is $M_{C}$.

4. The behaviour of the distribution function at the boundary $\Gamma_{r}$ of each valley is of the form

$$
\underbrace{\mathbf{k k} \ldots \mathbf{k}}_{M \text { times }} f_{r}(\mathbf{x}, \mathbf{k}, t) \rightarrow 0 \quad \text { as } \mathbf{k} \rightarrow \mathbf{k}\left(\Gamma_{r}\right) \text {. }
$$

This assumption simplifies the form of $\mathscr{G}_{M}$ because it eliminates a term when integrating by parts (the same result could be obtained by exploiting the periodicity of the crystal).

5. If the boundary conditions vary in time, their maximum frequency is much lower than $v_{r r}$. This makes it possible to neglect the time derivative $\partial \overline{\mathscr{K}}_{M r} / \partial t$ and assume a steady-state approximation. It is worth reminding that this argument does not apply to the case $M=0$, hence the time derivative must be retained there unless the boundary conditions are truly static.

To complete this section it is worth indicating the form of the moment of rank 0

$$
\frac{\partial n}{\partial t}+\operatorname{div}\left(n \mathbf{v}_{n}\right)=C_{n},
$$

representing the continuity equation for $n$. It is easily seen that all intervalley and intravalley transitions (collectively indicated as intraband transitions) cancel out in the derivation of (14), whose right hand side is thus made of the interband transitions 
only. This is in contrast to what happens in all the other moments (1) where, thanks to the simplifying hypothesis on the frequencies associated to the collision mechanisms, only the intravalley terms are retained.

\section{SOLUTION SCHEME}

The structure of the moments equations (1) lends itself to the adoption of a solution scheme which will be illustrated in this section. The aim is that of transforming each pair of two consecutive first-order PDEs into a second-order PDE. Since the grouping starts from the lowest-rank moment $M=0$, the pairs will be labeled $(0,1),(2,3), \ldots,(M, M+$ $1), \ldots$, with $M$ even. For the sake of simplicity, the steady-state case will be considered. It must be pointed out that the discussion here focuses on the form of the equations and, later on, on the closure condition. In this context it is no limitation to assume the simplification of a single, spherical and parabolic valley $E(\mathbf{k})$ centered in the origin of space k. The non-parabolicity of the band may be taken into account by embedding it into the coefficients, the latter to be determined by a direct solution of the BTE. This has been shown in [12] using the Monte Carlo method, and later on in [13] using the method based on the spherical-harmonic expansion [14]. The anisotropy of the band, in turn, is averaged when adding up the contributions of the valleys.

Letting $M_{C}=1, E(\mathbf{k})=\hbar^{2} k^{2} /(2 m)$, from the definition of the group velocity

$$
\mathbf{u}=\frac{1}{\hbar} \operatorname{grad}_{k} E(\mathbf{k})
$$

one finds

$$
\hbar \mathbf{k}=m \mathbf{u}=m\left(\mathbf{v}_{n}+\mathbf{c}_{n}\right) .
$$

In the above, $m$ is the (scalar) effective mass of the electron. From (4), (5), and (16) it is easily found

$$
n \mathbf{v}_{n}=\frac{\hbar}{m} \int_{B} \mathbf{k} f \mathrm{~d}^{3} k=\frac{\hbar}{m} n \overline{\mathscr{K}}_{1} .
$$

Letting $M_{C}=1$ in (3) and using a vector identity one gets

$$
\begin{aligned}
n \mathscr{V}_{M} & =n \mathbf{v}_{n} \cdot \operatorname{grad} \overline{\mathscr{K}}_{M} \\
& =\operatorname{div}\left(\overline{\mathscr{K}}_{M} n \mathbf{v}_{n}\right)-\overline{\mathscr{K}}_{M} \operatorname{div}\left(n \mathbf{v}_{n}\right)
\end{aligned}
$$

Using (14) to replace $\operatorname{div}\left(n \mathbf{v}_{n}\right)$ at the right hand side, and rearranging the terms in (1), one finds

$$
\begin{aligned}
&-\operatorname{div}(\left.n \mathscr{R}_{M+1}+n \overline{\mathscr{K}}_{M} \mathbf{v}_{n}\right) \\
& \quad=n \frac{q}{\hbar} \overline{\mathscr{G}}_{M}+n \mathcal{N}_{2 M} \cdot\left(\overline{\mathscr{K}}_{M}-\overline{\mathscr{K}}_{M}^{\mathrm{eq}}\right)-C_{n} \overline{\mathscr{K}}_{M} .
\end{aligned}
$$

Finally, replacing $M$ with $M+1$ in (1) and remembering that $\overline{\mathscr{K}}_{M+1}^{\text {eq }}=0$, one finds the second equation of the pair in the form

$$
\begin{gathered}
-n \mathscr{N}_{2 M+2} \cdot \overline{\mathscr{K}}_{M+1}-\frac{\hbar}{m} n \overline{\mathscr{K}}_{1} \cdot \operatorname{grad} \dot{\mathscr{\mathscr { K }}}_{M+1} \\
=\operatorname{div}\left(n \mathscr{R}_{M+2}\right)+n \frac{q}{\hbar} \overline{\mathscr{G}}_{M+1} .
\end{gathered}
$$

The second term at the left hand side of (20) is usually referred to as convective term. For $M=0$ it is

$$
\begin{gathered}
\overline{\mathscr{K}}_{0}=\overline{\mathscr{K}}_{0}^{\mathrm{eq}}=1, \quad \overline{\mathscr{G}}_{0}=0, \quad \overline{\mathscr{G}}_{1}=\mathbf{F}, \\
n \mathscr{R}_{1}=\bar{c}_{n}=0
\end{gathered}
$$

whence, remembering (17), the pair $(0,1)$ reads

$$
\begin{gathered}
\operatorname{div}\left(n \mathbf{v}_{n}\right)=C_{n} \\
-\mathscr{N}_{2} \cdot n \mathbf{v}_{n}-n \mathbf{v}_{n} \cdot \operatorname{grad} \mathbf{v}_{n} \\
=\operatorname{div}\left(\frac{\hbar}{m} n \mathscr{R}_{2}\right)+\frac{q}{m} n \mathbf{F} .
\end{gathered}
$$

Eq. (22) is of course the steady-state form of (14). As for (23), the second term at the left hand side (i.e., the convective term for $M=0$ ) is negligible when the velocity regime of the carriers is subsonic [15], which is typically the case of Silicon devices operating at room temperature. No indication is available in the literature, at least in the authors' knowledge, about the magnitude of the convective term of rank $M+1, M=2,4, \ldots$. It will be assumed here that in the subsonic regime the convective term is negligible for all odd moments; this hypothesis must of course be corroborated by a direct solution of the BTE. The transformation of the pair (19), (20) into a second-order equation is now easily accomplished if one notes that $n \overline{\mathscr{K}}_{M+1}$ is proportional to the argument of the divergence operator at the left hand side of (19). In fact, letting 
$M_{C}=1$ in (2), (4), (5), one gets

$$
\begin{aligned}
n \mathscr{R}_{M+1}+n \overline{\mathscr{K}}_{M} \mathbf{v}_{n} & =\int_{B} \mathscr{K}_{M} \mathbf{c}_{n} f \mathrm{~d}^{3} k+\int_{B} \mathscr{K}_{M} \mathbf{v}_{n} f \mathrm{~d}^{3} k \\
& =\int_{B} \mathscr{K}_{M} \mathbf{u} f \mathrm{~d}^{3} k
\end{aligned}
$$

Then, from (17),

$$
\begin{aligned}
\int_{B} \mathscr{K}_{M} \mathbf{u} f \mathrm{~d}^{3} k & =\frac{\hbar}{m} \int_{B} \mathscr{K}_{M+1} f \mathrm{~d}^{3} k \\
& =\frac{\hbar}{m} n \overline{\mathscr{K}}_{M+1} .
\end{aligned}
$$

Finally, extracting $\overline{\mathscr{K}}_{M+1}$ from (20) and replacing into (19), one finds ( $M$ even):

$$
\begin{gathered}
\operatorname{div}\left[\left(\mathscr{N}_{2 \dot{M}+2}\right)^{-1} \cdot\left\{\operatorname{div}\left(\frac{\hbar}{m} n \mathscr{R}_{M+2}\right)+\frac{q}{m} n \overline{\mathscr{G}}_{M+1}\right\}\right] \\
=n \frac{q}{\hbar} \overline{\mathscr{G}}_{M}+n \mathscr{N}_{2 M} \cdot\left(\overline{\mathscr{K}}_{M}-\overline{\mathscr{K}}_{M}^{\mathrm{eq}}\right)-C_{n} \overline{\mathscr{K}}_{M} .
\end{gathered}
$$

The terms of the above second-order equation must now be analyzed. To this purpose one admits that, when solving (26) for a given $M$, some quantities be available either from the solution at different $M$ s, or from the previous iterate of the same $M$ or, finally, from independent information (like Poisson's equation, the equilibrium regime, or the closure condition). By way of example, for $M=0$ the above becomes

$$
\begin{gathered}
\operatorname{div}\left[\left(\mathscr{N}_{2}\right)^{-1} \cdot\left\{\operatorname{div}\left(\frac{\hbar}{m} n \mathscr{R}_{2}\right)+\frac{q}{m} n \mathbf{F}\right\}\right]=-C_{n}, \\
\frac{\hbar}{m} n \mathscr{R}_{2}=\int_{B} \mathbf{c}_{n} \mathbf{c}_{n} f \mathrm{~d}^{3} k,
\end{gathered}
$$

where $\mathbf{F}$ and $\mathscr{R}_{2}$ are taken from Poisson's equation and from the moment of rank 2, respectively, and $C_{n}$ is expressed as a function of $n$. The inverse relaxation time $\mathscr{N}_{2}$, as discussed in section 2 , is approximated as a function of the moments themselves. Eq. (27) can then be solved for $n$, which is to be considered the primary unknown. The average velocity $\mathbf{v}_{n}$, which in this scheme is to be considered the secondary unknown, is finally recovered from (23). Turning now to the general case $M \geq 2$, it will be assumed that $\mathscr{N}_{2 M}, \mathscr{N}_{2 M+2}, \overline{\mathscr{R}}_{M}^{\text {eq }}, C_{n}$, and $\mathbf{F}$ are given in (26). The next step of the analysis consists
TABLE 1

Maximum rank of the tensor products $(M=2,4, \ldots)$.

\begin{tabular}{|c|c|c|c|c|}
\cline { 2 - 5 } \multicolumn{1}{c|}{} & $\overline{\mathscr{S}}_{M}$ & $\overline{\mathscr{S}}_{M+1}$ & $\overline{\mathscr{T}}_{M}$ & $\mathscr{R}_{M+2}$ \\
\hline$v_{n} \ldots v_{n}$ & $M-1$ & $M$ & $M$ & $M+1$ \\
\hline$c_{n} \ldots c_{n}$ & $M-1$ & $M$ & $M$ & $M+2$ \\
\hline
\end{tabular}

in replacing (16) in the remaining quantities $\overline{\mathscr{G}}_{M}, \overline{\mathscr{G}}_{M+1}, \overline{\mathscr{K}}_{M}, \mathscr{R}_{M+2}$, and evaluating the maximum rank of the tensor products of the form $\mathrm{v}_{n} \ldots \mathrm{v}_{n}$ and $\mathbf{c}_{n} \ldots \mathbf{c}_{n}$ appearing in each of them. The result is summarized in Table 1. The mixed products such as $\mathbf{v}_{n} c_{n} \ldots$ are not reported for the sake of conciseness. Now, all products of the form $\mathbf{v}_{n} \ldots \mathbf{v}_{n}$ are given since $\mathbf{v}_{n}$ is taken from the solution of the pair $(0,1)$. All mixed products $\mathbf{v}_{n} \mathbf{c}_{n} \ldots$ are given as well: in fact, the number of $\mathbf{c}_{n}$ s here is smaller than $M$, hence they form the unknowns of lower-rank equations. The remaining products are of the form $\mathbf{c}_{n} \ldots \mathbf{c}_{n}$. That of rank $M$ (appearing in $\overline{\mathscr{G}}_{M+1}, \overline{\mathscr{K}}_{M}$, and $\mathscr{R}_{M+2}$ ) must be taken as primary unknown, while that of rank $M+1$ (appearing in $\mathscr{R}_{M+2}$ ) forms a part of the secondary unknown; finally, the product of rank $M+2$, which also appears in $\mathscr{R}_{M+2}$, is a higher-rank unknown which links the current pair $(M, M+1)$ to the next one. Comparing with (27), one sees that product $\mathbf{c}_{n} \mathbf{c}_{n}$ constitutes such a link there. It is also worth observing that no contribution of rank 1 stems from $\mathscr{R}_{2}$; this is a special case and is due to the fact that $\bar{c}_{n}=0$.

The above discussion lends itself to the determination of a closure condition which is intrinsic to the scheme itself. To this purpose it is useful to base the reasoning on (20). There one sees in fact that the "link" appears at the right hand side within the argument of the divergence operator. The secondary unknown, in turn, is present at both sides, namely, in algebraic form on the left and within the divergence operator on the right. The idea is that of obtaining the closure by neglecting the divergence of terms of rank higher than $M$ in $\mathbf{c}_{n}$. To accomplish this one must use (2), (5), and (16) to get

$$
\begin{aligned}
n \mathscr{R}_{M+2}= & \left(\frac{m}{\hbar}\right)^{M+1} \\
& \times \int_{B} \frac{\left(\mathbf{v}_{n}+\mathbf{c}_{n}\right) \ldots\left(\mathbf{v}_{n}+\mathbf{c}_{n}\right)}{M+1 \text { factors }} \mathbf{c}_{n} f \mathrm{~d}^{3} k .
\end{aligned}
$$

The terms to be neglected can be grouped to form 
two tensors, namely

$$
n \mathscr{E}_{M+2}=\left(\frac{m}{\hbar}\right)^{M+1} \int_{B} \underbrace{\mathbf{c}_{n} \ldots \mathbf{c}_{n}}_{M+2 \text { factors }} f \mathrm{~d}^{3} k
$$

and

$$
\begin{aligned}
& n \mathscr{F}_{M+2}=\left(\frac{m}{\hbar}\right)^{M+1} \\
& \times \int_{B} \underbrace{}_{\left(\mathbf{v}_{n} \mathbf{c}_{n} \mathbf{c}_{n} \ldots \mathbf{c}_{n}+\mathbf{c}_{n} \mathbf{v}_{n} \mathbf{c}_{n} \ldots \mathbf{c}_{n}+\cdots+\mathbf{c}_{n} \ldots \mathbf{c}_{n} \mathbf{c}_{n} \mathbf{v}_{n}\right)} \mathbf{c}_{n} f \mathrm{~d}^{3} k \\
& M+1 \text { terms of } M+1 \text { factors each }
\end{aligned}
$$

The closure condition then reads

$$
\operatorname{div}\left(\mathscr{E}_{M+2}+\mathscr{F}_{M+2}\right)=0 .
$$

It is worth noting that the closure condition proposed above involves only the sum of the derivatives of a few unknowns. This must be regarded as an advantage since it is likely to limit the perturbation induced by the closure condition onto the system to be solved. On the other hand, an expression in divergence form is in itself insufficient to fully determine the argument $\mathscr{E}_{M+2}+\mathscr{F}_{M+2}$. It follows that specifying further conditions is necessary when such an argument must be calculated. This will be discussed in a few examples of section 4. Another observation is that, due to definition (29), the link between the current pair and the next one would be eliminated by prescribing the divergence of $\mathscr{E}_{M+2}$ only. Neglecting the divergence of $\mathscr{F}_{M+2}$ as well has the advantage of rendering (20) algebraic in the secondary unknown, thus simplifying somewhat the solution of (26).

\section{EXAMPLES}

The theory depicted in section 3 will be applied here to two special cases. In the first one, the system to be solved is made of the pair $(0,1)$ only and leads to (22), (23) or, equivalently, to (27). Comparing (29), (30) with the second of (27) one finds $\mathscr{E}_{2}=\mathscr{R}_{2}$, $\mathscr{F}_{2}=0$, whence the closure condition (31) reduces to $\operatorname{div} \mathscr{R}_{2}=0$. It is customary to introduce a rank 2 tensor $\mathscr{T}_{n}$ such that

$$
n k_{B} \mathscr{T}_{n}=\hbar n \mathscr{R}_{2}=m \int_{B} \mathbf{c}_{n} \mathbf{c}_{n} f \mathrm{~d}^{3} k
$$

where $k_{B}$ is Boltzmann's constant. Tensor $\mathscr{T}_{n}$ is dimensionally a temperature and is referred to as temperature tensor of the electrons. The closure condition can then be rewritten as $\operatorname{div} \mathscr{T}_{n}=0$. In the typical operating conditions the departure of $\mathscr{T}_{n}$ from the scalar form is negligible [10], such that one may assume $\mathscr{T}_{n}=T_{n} \mathscr{I}_{2}$ and the closure condition becomes $\operatorname{grad} T_{n}=0$. The electron temperature then reduces to a constant $T_{n}^{\text {eq }}$ which, due to the boundary conditions, is set equal to the lattice temperature. As for the left hand side of (23), one must now evaluate the inverse macroscopic relaxation time $\mathscr{N}_{2}$. In principle, this tensor could be calculated in terms of the microscopic quantities [1]. In the frame of the simplified model discussed here, however, it is customary to reduce it to a scalar by letting $\mathscr{N}_{2}=$ $\left(1 / \tau_{p n}\right) \mathscr{I}_{2}$. The quantity $\tau_{p n}>0$ is called momentum-relaxation time and is determined experimentally. Letting

$$
\begin{gathered}
\mu_{n}=\frac{q}{m} \tau_{p n}, \quad D_{n}^{\mathrm{eq}}=\frac{k_{B} T_{n}^{\mathrm{eq}}}{q} \mu_{n}, \\
\mathbf{J}_{n}=-q n \mathbf{v}_{n}, \quad U=-q C_{n},
\end{gathered}
$$

(22), (23) take the form

$\operatorname{div} \mathbf{J}_{n}=q U, \quad \mathbf{J}_{n}=q \mu_{n} n \mathbf{F}+q D_{n}^{\mathrm{eq}} \operatorname{grad} n$.

The first two quantities defined in (33) are called mobility and diffusivity of the electrons, respectively. The third one is the electron-current density. The last one is called net recombination rate. Due to its form, the model defined by (34) is referred to as drift-diffusion model. In the second case to be considered, the system is made of the two pairs $(0,1)$, and $(2,3)$. The first one leads to (22), (23) while the second, letting $M=2$ in (19) and (20) and neglecting the convective term, yields for us

$$
\begin{aligned}
-\operatorname{div}( & \left.n \mathscr{R}_{3}+n \overline{\mathscr{K}}_{2} \mathbf{v}_{n}\right) \\
= & n \frac{q}{\hbar} \overline{\mathscr{G}}_{2}+n \mathscr{N}_{4} \cdot\left(\overline{\mathscr{K}}_{2}-\overline{\mathscr{K}}_{2}^{\mathrm{eq}}\right)-C_{n} \overline{\mathscr{K}}_{2}, \\
& -n \mathscr{N}_{6} \cdot \overline{\mathscr{K}}_{3}=\operatorname{div}\left(n \mathscr{R}_{4}\right)+n \frac{q}{\hbar} \overline{\mathscr{G}}_{3} .
\end{aligned}
$$

One finds

$$
n \overline{\mathscr{K}}_{2}=\left(\frac{m}{\hbar}\right)^{2} \int_{B}\left(\mathbf{v}_{n}+\mathbf{c}_{n}\right)\left(\mathbf{v}_{n}+\mathbf{c}_{n}\right) f \mathrm{~d}^{3} k
$$


which, thanks to (32), yields

$$
\overline{\mathscr{K}}_{2}=\frac{m}{\hbar^{2}}\left(m \mathbf{v}_{n} \mathbf{v}_{n}+k_{B} \mathscr{T}_{n}\right)=\frac{2 m}{\hbar^{2}} \mathscr{W}_{n} .
$$

Tensor $\mathscr{W}_{n}=\left(m \mathbf{v}_{n} \mathbf{v}_{n}+k_{B} \mathscr{T}_{n}\right) / 2$ is dimensionally an energy and is referred to as energy tensor of the electrons. For the drift terms one finds

$$
n \frac{q}{\hbar} \overline{\mathscr{G}}_{2}=q n \frac{m}{\hbar^{2}}\left(\mathbf{F} \mathbf{v}_{n}+\mathbf{v}_{n} \mathbf{F}\right)
$$

in (35) and

$$
\begin{aligned}
n \frac{q}{\hbar} \overline{\mathscr{G}}_{3}= & q \frac{m^{2}}{\hbar^{3}}\left[n\left(\mathbf{F v}_{n} \mathbf{v}_{n}+\mathbf{v}_{n} \mathbf{F} \mathbf{v}_{n}+\mathbf{v}_{n} \mathbf{v}_{n} \mathbf{F}\right)\right. \\
& \left.+\int_{B}\left(\mathbf{F c}_{n} \mathbf{c}_{n}+\mathbf{c}_{n} \mathbf{F c}_{n}+\mathbf{c}_{n} \mathbf{c}_{n} \mathbf{F}\right) f \mathrm{~d}^{3} k\right]
\end{aligned}
$$

in (36). The tensors defining the closure condition are found from (29), (30) as

$$
n \mathscr{E}_{4}=\left(\frac{m}{\hbar}\right)^{3} \int_{B} \mathbf{c}_{n} \mathbf{c}_{n} \mathbf{c}_{n} \mathbf{c}_{n} f \mathrm{~d}^{3} k
$$

and

$$
n \mathscr{F}_{4}=\left(\frac{m}{\hbar}\right)^{3} \int_{B}\left(\mathbf{v}_{n} \mathbf{c}_{n} \mathbf{c}_{n}+\mathbf{c}_{n} \mathbf{v}_{n} \mathbf{c}_{n}+\mathbf{c}_{n} \mathbf{c}_{n} \mathbf{v}_{n}\right) \mathbf{c}_{n} f \mathrm{~d}^{3} k .
$$

Taking into account the fact that $\bar{c}_{n}=0$, the remaining part of $n \mathscr{R}_{4}$ we find to be

$$
\begin{aligned}
n \mathscr{H}_{4} & =n\left(\mathscr{R}_{4}-\mathscr{E}_{4}-\mathscr{F}_{4}\right) \\
& =\left(\frac{m}{\hbar}\right)^{3} \int_{B}\left(\mathbf{v}_{n} \mathbf{v}_{n} \mathbf{c}_{n}+\mathbf{v}_{n} \mathbf{c}_{n} \mathbf{v}_{n}+\mathbf{c}_{n} \mathbf{v}_{n} \mathbf{v}_{n}\right) \mathbf{c}_{n} f \mathrm{~d}^{3} k .
\end{aligned}
$$

Finally, due to (31), the first term on the right hand side of (36) simplifies to

$$
\begin{aligned}
\operatorname{div}\left(n \mathscr{R}_{4}\right)= & n \operatorname{div}\left(\mathscr{H}_{4}\right) \\
& +\left(\mathscr{E}_{4}+\mathscr{F}_{4}+\mathscr{H}_{4}\right) \cdot \operatorname{grad} n .
\end{aligned}
$$

The tensor nature of the above equations is still quite complicated when considering the problem of their solution in realistic cases. Further manipulations are then introduced to decrease the tensors' rank. First, the already mentioned simplifications $\mathscr{T}_{n}=T_{n} \mathscr{I}_{2}, \mathscr{N}_{2}=\left(1 / \tau_{p n}\right) \mathscr{I}_{2}$ are kept, whence (22),
(23) become

$$
\operatorname{div} \mathbf{J}_{n}=q U, \quad \mathbf{J}_{n}=q \mu_{n} n \mathbf{F}+q D_{n}^{\text {eq }} \operatorname{grad}\left(n T_{n} / T_{n}^{\text {eq }}\right) .
$$

In the above, the symbols are those defined in (33). For pair $(2,3)$ the simplifications are achieved in a similar manner by letting $\mathscr{N}_{4}=\left(1 / \tau_{w n}\right) \mathscr{I}_{4}, \mathscr{N}_{6}=$ $\left(1 / \tau_{q n}\right) \mathscr{f}_{6}\left(\tau_{w n}, \tau_{q n}>0\right)$. This approximation is expected to hold in realistic devices because the carriers can be regarded as a slowly drifting gas [12]. Then, the trace only of (35) is considered; from (38) and (39) one finds

$$
\begin{aligned}
\operatorname{tr}\left(\overline{\mathscr{K}}_{2}\right) & =\frac{m}{\hbar^{2}}\left(m v_{n}^{2}+3 k_{B} T_{n}\right)=\frac{2 m}{\hbar^{2}} w_{n}, \\
\operatorname{tr}\left(n \frac{q}{\hbar} \overline{\mathscr{G}}_{2}\right) & =q n \frac{2 m}{\hbar^{2}} \mathbf{F} \cdot \mathbf{v}_{n}=-\frac{2 m}{\hbar^{2}} \mathbf{F} \cdot \mathbf{J}_{n},
\end{aligned}
$$

where $w_{n}$ is the average kinetic energy of the electrons, defined as

$$
\begin{gathered}
w_{n}=\operatorname{tr}\left(\mathscr{W}_{2}\right)=\frac{1}{2} m v_{n}^{2}+\frac{3}{2} k_{B} T_{n}, \\
w_{n}^{\mathrm{eq}}=\operatorname{tr}\left(\mathscr{W}_{2}^{\mathrm{eq}}\right)=\frac{3}{2} k_{B} T_{n}^{\mathrm{eq}}
\end{gathered}
$$

Thanks to (46), the right hand side of (35) yields a scalar. The manipulation of the left hand side becomes easier if one remembers that for (24), (25) it is $n \mathscr{R}_{3}+n \overline{\mathscr{K}}_{2} \mathbf{v}_{n}=(\hbar / m) n \overline{\mathscr{K}}_{3}$, whence

$$
\begin{aligned}
\operatorname{tr}\left[\operatorname{div}\left(n \mathscr{R}_{3}+n \overline{\mathscr{K}}_{2} \mathbf{v}_{n}\right)\right] \\
=\frac{\hbar}{m} \operatorname{tr}\left[\operatorname{div} \int_{B} \mathbf{k k k} f \mathrm{~d}^{3} k\right] \\
=\frac{\hbar}{m} \sum_{j} \frac{\partial}{\partial x_{j}} \int_{B} f k_{j} \sum_{i} k_{i}^{2} \mathrm{~d}^{3} k \\
=\left(\frac{m}{\hbar}\right)^{2} \operatorname{div} \int_{B}\left(v_{n}^{2}+c_{n}^{2}+2 \mathbf{v}_{n} \cdot \mathbf{c}_{n}\right) \\
\\
\quad\left(\mathbf{v}_{n}+\mathbf{c}_{n}\right) f \mathrm{~d}^{3} k .
\end{aligned}
$$

Observing that $\left(\mathbf{v}_{n} \cdot \mathbf{c}_{n}\right) \mathbf{c}_{n}=\left(\mathbf{c}_{n} \mathbf{c}_{n}\right) \cdot \mathbf{v}_{n}$ and defining a vector

$$
\mathbf{P}_{n}=\left(w_{n}+k_{B} T_{n}\right) \mathbf{v}_{n}+\frac{m}{2} \overline{c_{n}^{2} \mathbf{c}_{n}},
$$


the last term of (48) we find transforms into

$$
\left(2 m / \hbar^{2}\right) \operatorname{div}\left(n \mathbf{P}_{n}\right) \text {. }
$$

Eq. (35) then becomes

$$
\operatorname{div}\left(n \mathbf{P}_{n}\right)=C_{n} w_{n}-n \frac{w_{n}-w_{n}^{\mathrm{eq}}}{\tau_{w n}}+\mathbf{F} \cdot \mathbf{J}_{n} .
$$

Vector $\mathbf{P}_{n}$ is dimensionally an energy times a velocity and may be thought of as an energy flux. In turn, the three terms on the right hand side of (50) are energies per unit volume and time. The first one accounts for the variation in the average kinetic energy of the electrons due to the interband transitions, the second for that due to the intervalley transitions. The third one, which accounts for the variation due to the electric field, is usually referred to as Joule heating. The denominator $\tau_{w n}$ of the second term is called energy-relaxation time of the electrons. It is worth observing that the definition - and symbol as well - of the energy flux differ from that given in [1] (Eq. (133)), where the convective part of the energy was kept separated from the other terms. The definition given here must be preferred because it is consistent with the general structure of the solution method (see (24), (25)).

Turning now to (36) and remembering the simplification on the inverse relaxation time, one finds for the left hand side the expression $-\left(2 m^{2} / \hbar^{3}\right) n \mathbf{P}_{n} / \tau_{q n}$. The trace of the second term on the right hand side is easily found by observing that, due to symmetry, the sum can be carried out via any two vectors of the triplets, hence

$\operatorname{tr}\left(\frac{q}{\hbar} \overline{\mathscr{G}}_{3}\right)=\frac{2 m}{\hbar^{3}} n\left[\left(w_{n}+k_{B} T_{n}\right) q \mathbf{F}+m\left(\mathbf{v}_{n} \mathbf{v}_{n}\right) \cdot q \mathbf{F}\right]$.

The last part to be treated is the first term on the right hand side of (36), given by (44). It is worth remembering that, since the closure expression (31) involves the divergence only, further information must be supplemented in order to fully specify the sum $\mathscr{E}_{4}+\mathscr{F}_{4}$ appearing in (44). For instance, in the drift-diffusion model it was taken $\mathscr{T}_{n}=T_{n} \mathscr{I}_{2}$. For consistency a similar condition will be used here, that is, $\mathscr{E}_{4}+\mathscr{F}_{4}=\beta_{n} \mathscr{Y}_{4}$. This assumption can be corroborated by the same argument used above to introduce the scalar relaxation times. From the closure condition $\operatorname{div}\left(\beta_{n} \mathscr{I}_{4}\right)=\beta_{n} \operatorname{div} \mathscr{I}_{4}+\mathscr{I}_{4} \cdot \operatorname{grad} \beta_{n}$ $=0$ it then follows $\beta_{n}=$ const. The right hand side of (44), in turn, simplifies to $\operatorname{div}\left[n\left(\mathscr{H}_{4}+\beta_{n} \mathscr{Y}_{4}\right)\right]$. To calculate its trace, care must be taken because of the asymmetry introduced by the last vector $\mathbf{c}_{n}$ to the right in the definition (43) of $n \mathscr{H}_{4}$. To this purpose, it is worth observing that the divergences have always been calculated as $\operatorname{div} \mathscr{Z} \cdot \nabla$, i.e., with the derivative operator acting from right to left over the last index of the tensor's entries. For consistency, one must then use the last $\mathbf{c}_{n}$ to the right in (43) for calculating the divergence, and any two vectors of the triplets in the symmetrical sum in parenthesis to calculate the trace. Observing that $\left(\mathbf{c}_{n} \cdot \mathbf{v}_{n}\right) \mathbf{v}_{n} \mathbf{c}_{n}=\left(\mathbf{v}_{n} \mathbf{v}_{n}\right) \cdot\left(\mathbf{c}_{n} \mathbf{c}_{n}\right)$ and letting $\beta_{n}=$ $(2 / 3) \gamma_{n}\left(m / \hbar^{3}\right)\left(k_{B} T_{n}^{\text {eq }}\right)^{2}\left(\gamma_{n}\right.$ a number $)$, one gets

$$
\begin{aligned}
\operatorname{tr} \operatorname{div}\left(n \mathscr{H}_{4}+\beta_{n} n \mathscr{F}_{4}\right) & \\
= & \frac{2 m}{\hbar^{3}} \operatorname{div}\left[n k_{B} T_{n}\left(\frac{1}{2} m v_{n}^{2} \mathscr{I}_{2}+m \mathbf{v}_{n} \mathbf{v}_{n}\right)\right] \\
& +\frac{2 m}{\hbar^{3}} \gamma_{n}\left(k_{B} T_{n}^{\text {eq }}\right)^{2} \operatorname{grad} n .
\end{aligned}
$$

Combining with (51), the second equation of pair $(2,3)$ finally results in

$$
\begin{aligned}
-\frac{m}{\tau_{q n}} n \mathbf{P}_{n}= & \operatorname{div}\left[n k_{B} T_{n}\left(\frac{1}{2} m v_{n}^{2} \mathscr{J}_{2}+m \mathbf{v}_{n} \mathbf{v}_{n}\right)\right] \\
& +\gamma_{n}\left(k_{B} T_{n}^{\mathrm{eq}}\right)^{2} \operatorname{grad} n \\
& +n\left[\left(w_{n}+k_{B} T_{n}\right) q \mathbf{F}+m\left(\mathbf{v}_{n} \mathbf{v}_{n}\right) \cdot q \mathbf{F}\right] .
\end{aligned}
$$

In this way, the energy flux $\mathbf{P}_{n}$ (in which, due to the last term of (49), the maximum rank of the tensor products is 3) has been expressed in terms of the primary unknown $T_{n}$ (rank 2) and of the lower-rank quantities $n$ and $\mathbf{v}_{n}$. The denominator $\tau_{q n}$ of the left hand side of (53) can be thought of as the relaxation time associated to the energy flux. One sees that (53) is rather more complicated than the usual Fourier law by which the standard hydrodynamic model is typically closed. For this reason, the model constituted by (45), (50), and (53) is to be regarded as a more complete form of the hydrodynamic model. In equilibrium, $\mathbf{v}_{n}$ and $\mathbf{P}_{n}$ vanish. However, if the doping in the semiconductor is non-uniform it is still grad $n \neq 0$. Eqs. (45), (53) then reduce, respectively, to

$$
\begin{aligned}
k_{B} T_{n}^{\mathrm{eq}} \operatorname{grad} n & =-q n \mathbf{F}, \\
\frac{2}{5} \gamma_{n} k_{B} T_{n}^{\mathrm{eq}} \operatorname{grad} n & =-q n \mathbf{F},
\end{aligned}
$$


whence $\gamma_{n}=5 / 2$. Further discussion will be carried out in section 6 . Here it is worth adding that, in the standard operating mode, (53) can somewhat be simplified. The thermal part $(3 / 2) k_{B} T_{n}$ of the average kinetic energy is in fact dominant with respect to the drift part $(1 / 2) m v_{n}^{2}$. Using the same argument as the one adopted to simplify $\mathscr{T}_{n}, \mathscr{N}_{2}, \mathscr{N}_{4}$, and $\mathscr{N}_{6}$, we neglect $\mathbf{v}_{n} \mathbf{v}_{n}$ with respect to its trace, to find

$$
\begin{aligned}
-\frac{m}{\tau_{q n}} n \mathbf{P}_{n} \simeq & \operatorname{grad}\left[n\left(\frac{1}{2} m v_{n}^{2} k_{B} T_{n}+\frac{5}{2}\left(k_{B} T_{n}^{\mathrm{eq}}\right)^{2}\right)\right] \\
& +n \frac{5}{2} k_{B} T_{n} q \mathbf{F} .
\end{aligned}
$$

\section{THE COEFFICIENTS IN THE HD AND ET MODELS}

Starting from the general expressions of [1], the coefficients

$\mathscr{N}_{2}=\frac{1}{\tau_{p n}} \mathscr{I}_{2}, \quad \mathscr{N}_{4}=\frac{1}{\tau_{w n}} \mathscr{I}_{4}, \quad \mathscr{N}_{6}=\frac{1}{\tau_{q n}} \mathscr{I}_{6}$

of the hydrodynamic model can now be written in terms of the moments introduced in section 4, and of other integrals involving the microscopic relaxation time $\tau=1 / \nu$ of the BTE. The latter has been introduced in (10) and is considered here for a single valley. The starting point is Eq. (101) of [1], which reads

$$
\mathscr{N}_{2 M} \cdot\left(\overline{\mathscr{K}}_{M}-\tilde{\mathscr{\mathscr { K }}}_{M}\right)=\mathscr{N}_{2 M} \cdot\left(\overline{\mathscr{K}}_{M}-\overline{\mathscr{K}}_{M}^{\mathrm{eq}}\right),
$$

supplemented with the definitions

$$
\overline{\mathscr{K}}_{M}=\frac{1}{n} \int_{B} \mathscr{K}_{M} f \mathrm{~d}^{3} k, \quad \overline{\mathscr{K}}_{M}^{\mathrm{eq}}=\frac{1}{n^{\mathrm{eq}}} \int_{B} \mathscr{K}_{M} f^{\mathrm{eq}} \mathrm{d}^{3} k
$$

and

$$
\begin{aligned}
& \mathscr{N}_{2 M} \cdot \overline{\mathscr{K}}_{M}=\frac{1}{n} \int_{B} \mathscr{K}_{M} \nu f \mathrm{~d}^{3} k, \\
& \mathscr{N}_{2 M} \cdot \tilde{\mathscr{\mathscr { K }}}_{M}=\frac{1}{n} \int_{B} \mathscr{K}_{M} \nu \tilde{f} \mathrm{~d}^{3} k .
\end{aligned}
$$

Inserting (56), (58), (59) into (57) one finds the general expression

$$
\begin{gathered}
\frac{1}{n} \int_{B} \mathscr{K}_{M} f \mathrm{~d}^{3} k-\frac{1}{n^{\mathrm{eq}}} \int_{B} \mathscr{K}_{M} f^{\mathrm{eq}} \mathrm{d}^{3} k \\
=\tau_{2 M} \frac{1}{n} \int_{B} \mathscr{K}_{M} \frac{f-\tilde{f}}{\tau} \mathrm{d}^{3} k
\end{gathered}
$$

whence, letting $M=2$, we obtain

$$
\begin{gathered}
\frac{1}{n} \int_{B} \mathbf{k k} f \mathrm{~d}^{3} k-\frac{1}{n^{\mathrm{eq}}} \int_{B} \mathbf{k k} f^{\mathrm{eq}} \mathrm{d}^{3} k \\
=\tau_{w n} \frac{1}{n} \int_{B} \mathbf{k} \mathbf{k} \frac{f-\tilde{f}}{\tau} \mathrm{d}^{3} k .
\end{gathered}
$$

while, letting $M=1$ and $M=3$, and remembering that $f^{\text {eq }}(\mathbf{k})$ is even, we obtain

$$
\begin{gathered}
\int_{B} \mathbf{k} f \mathrm{~d}^{3} k=\tau_{p n} \int_{B} \mathbf{k} \frac{f-\tilde{f}}{\tau} \mathrm{d}^{3} k, \\
\int_{B} \mathbf{k k k} f \mathrm{~d}^{3} k=\tau_{q n} \int_{B} \mathbf{k k k} \frac{f-\tilde{f}}{\tau} \mathrm{d}^{3} k .
\end{gathered}
$$

Assuming now that the valley is spherical and parabolic, and taking the trace of (61), one finds

$$
n\left(w_{n}-w_{n}^{\mathrm{eq}}\right)=\tau_{w n} \int_{B} \frac{1}{2} m u^{2} \frac{f-\tilde{f}}{\tau} \mathrm{d}^{3} k .
$$

Similarly, from (62),

$$
\begin{aligned}
n \mathbf{v}_{n} & =\tau_{p n} \int_{B} \mathbf{u} \frac{f-\tilde{f}}{\tau} \mathrm{d}^{3} k \\
n \mathbf{P}_{n} & =\tau_{q n} \int_{B} \frac{1}{2} m u^{2} \mathbf{u} \frac{f-\tilde{f}}{\tau} \mathrm{d}^{3} k .
\end{aligned}
$$

Remembering the general expression (10) of the scattering term and the meaning of the right hand side of (14), one sees that the scattering processes incorporated in $\tilde{f}$ of (63)-(64) are the intraband transitions (typical examples are phonon, impurity, and electron-electron scattering). The interband transitions, in turn, are embedded in $C_{n}$ of (14). Examples of application of the hydrodynamic model where $C_{n}$ accounts for Shockley-Read-Hall, Auger, and impact-ionization transitions are given, e.g., in $[6,16]$. It will be shown in section 6 that the ratio $\tau_{q n} / \tau_{p n}$ appears in the expression of the heat flux and thermal conductivity. The two relaxation times have been calculated by the method of [14] and are shown in Fig. 1. 


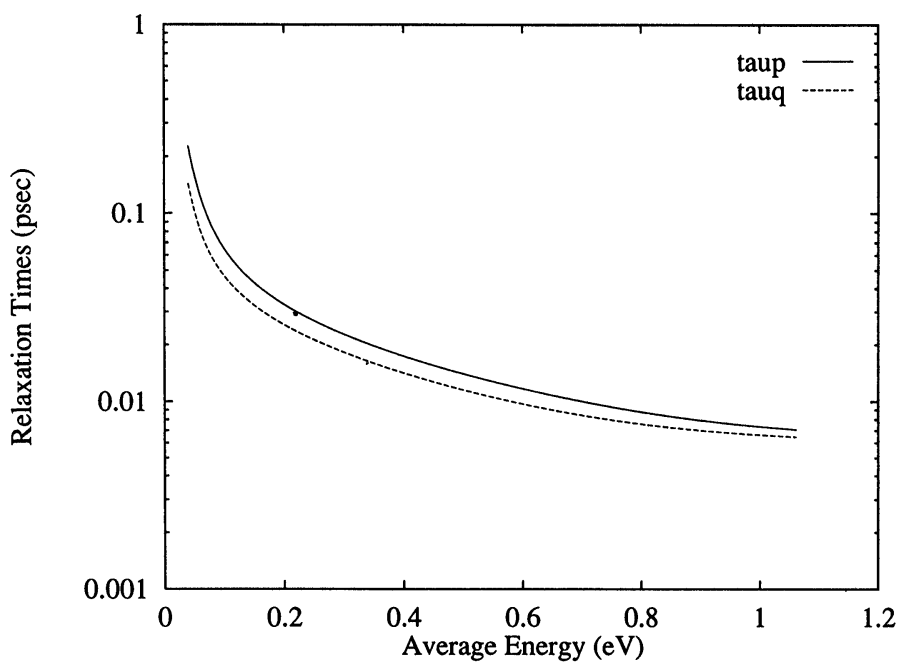

FIGURE 1 Electron momentum-relaxation time $\left(\tau_{p n}\right)$ and heat-relaxation time $\left(\tau_{q n}\right)$ as a function of the average energy.

In recent years, a transport model involving the moments up to order 2 of the BTE has been proposed, which is referred to as energy-transport model [8]. In the following, the features of the energytransport model will be re-examined within the general frame of the moment method. It will be shown that, although the form of the equations looks similar to that of the hydrodynamic model, more complications are actually hidden in the coefficients of the odd moments. Since the analysis is similar for the carrier- and energy-continuity equations, for the sake of simplicity the $M=1$ case only will be considered. The corresponding moment turns out to be [1]

$$
\begin{aligned}
-\int_{B} \mathbf{u} f_{1} \mathrm{~d}^{3} k= & \int_{B} \tau \mathbf{u}\left(\mathbf{u} \cdot \operatorname{grad}_{x} f_{0}\right) \mathrm{d}^{3} k \\
& -\frac{q}{\hbar} \int_{B} \tau \mathbf{u}\left(\mathbf{F} \cdot \operatorname{grad}_{k} f_{0}\right) \mathrm{d}^{3} k,
\end{aligned}
$$

where

$$
f_{0}=\langle f\rangle=\frac{1}{4 \pi} \int_{0}^{2 \pi} \int_{0}^{\pi} f \sin \vartheta \mathrm{d} \vartheta \mathrm{d} \varphi
$$

is the average value of the distribution function on a surface at constant $k$ in the $\mathbf{k}$ space, with $\mathrm{d} \Omega=$ $\sin \vartheta \mathrm{d} \vartheta \mathrm{d} \varphi$ the elementary solid angle, and

$$
f_{1}=f-f_{0} .
$$

Introducing the diagonal tensors

$$
n\left(\mathscr{A}_{n}\right)_{i i}=-q \int_{B} \frac{\partial f_{0}}{\partial E} \tau u_{i}^{2} \mathrm{~d}^{3} k
$$

and

$$
n\left(\mathscr{B}_{n}\right)_{i i}=\int_{B} f_{0} u_{i}^{2} \tau \mathrm{d}^{3} k, \quad n\left(\mathbf{h}_{n}\right)_{i}=\int_{B} f_{0} u_{i}^{2} \frac{\partial \tau}{\partial x_{i}} \mathrm{~d}^{3} k
$$

(65) becomes

$$
-n \mathbf{v}_{n}=n \mathscr{A}_{n} \cdot \mathbf{F}+\operatorname{div}\left(n \mathscr{B}_{n}\right)-n \mathbf{h}_{n}
$$

Assuming that $f_{0}$ has enough symmetry to transform $\mathscr{A}_{n}$ and $\mathscr{B}_{n}$ into scalars, (68) and the first equation of (69) yield

$$
n \mu_{n}^{\mathrm{et}}=-\frac{q}{3} \int_{B} \frac{\partial f_{0}}{\partial E} \tau u^{2} \mathrm{~d}^{3} k, \quad n B_{n}=\frac{1}{3} \int_{B} f_{0} u^{2} \tau \mathrm{d}^{3} k
$$

Introducing the definitions $\mathbf{J}_{\mathrm{n}}^{\mathrm{et}}=-q n \mathbf{v}_{n}, \mathbf{J}_{n h}^{\mathrm{et}}=$ $-q n \mathbf{h}_{n}$, (70) yields a transport equation of the form

$$
\mathbf{J}_{n}^{\mathrm{et}}=q \mu_{n}^{\mathrm{et}} n \mathbf{F}+q \operatorname{grad}\left(B_{n} n\right)+\mathbf{J}_{n h}^{\mathrm{et}} .
$$

It may be observed that the only term containing the electric field in (72) in $q \mu_{n}^{\text {et }} n \mathbf{F}$. Hence, the definition (71) of mobility in the energy-transport model is readily compared with the corresponding one in the hydrodynamic model, given by $q \tau_{p n} / m$ with $\tau_{p n}$ given by (64). As far as quantity $B_{n}$ in (71) is concerned, the standard derivation of the model relates it to the carrier temperature. It should be pointed out, however, that $B_{n}$ contains the convective term as well, as can be shown by splitting the group velocity in the 
usual way, i.e. $\mathbf{u}=\mathbf{c}_{n}+\mathbf{v}_{n}$ :

$$
\begin{aligned}
n B_{n} & =\frac{1}{3} \int_{B} f_{0}\left(c_{n}^{2}+v_{n}^{2}+2 \mathbf{c}_{n} \cdot \mathbf{v}_{n}\right) \tau \mathrm{d}^{3} k \\
& =\frac{1}{3} \int_{B} f_{0} c_{n}^{2} \tau \mathrm{d}^{3} k+\frac{1}{3} v_{n}^{2} \int_{B} f_{0} \tau \mathrm{d}^{3} k
\end{aligned}
$$

If we set

$$
\mu_{n c}^{\mathrm{et}}=\frac{q}{m} \frac{\int_{B} f_{0} c_{n}^{2} \tau \mathrm{d}^{3} k}{\int_{B} f_{0} c_{n}^{2} \mathrm{~d}^{3} k}, \quad T_{n}^{\mathrm{et}}=\frac{m}{3 k_{B}} \frac{\int_{B} f_{0} c_{n}^{2} \mathrm{~d}^{3} k}{\int_{B} f_{0} \mathrm{~d}^{3} k}
$$

the first integral at the right hand side of (73) transforms into

$$
\frac{1}{3} \int_{B} f_{0} c_{n}^{2} \tau \mathrm{d}^{3} k=n \mu_{n c}^{\mathrm{et}} \frac{k_{B} T_{n}^{\mathrm{et}}}{q}
$$

while letting

$$
\tilde{\tau}=\frac{1}{3} \frac{\int_{B} f_{0} \tau \mathrm{d}^{3} k}{\int_{B} f_{0} \mathrm{~d}^{3} k}
$$

transforms the second integral into

$$
\frac{1}{3} v_{n}^{2} \int_{B} f_{0} \tau \mathrm{d}^{3} k=n \tilde{\tau} v_{n}^{2}
$$

By replacing (75), (77) into (72), and neglecting the convective term $q \tilde{\tau} n v_{n} \operatorname{grad} v_{n}$, the transport equation in the energy-transport model takes the form

$$
\begin{aligned}
\mathbf{J}_{n}^{\text {et }}= & q \mu_{n}^{\text {et }} n \mathbf{F}+n \operatorname{grad}\left(\mu_{n c}^{\text {et }} k_{B} T_{n}^{\text {et }}\right) \\
& +\mu_{n c}^{\text {et }} k_{B} T_{n}^{\text {et }} \operatorname{grad} n+q v_{n} \operatorname{grad}\left(\tilde{\tau} n v_{n}\right)+\mathbf{J}_{n h}^{\text {et }},
\end{aligned}
$$

to be compared with the hydrodynamic one (45)

$$
\mathbf{J}_{n}=q \mu_{n} n \mathbf{F}+\mu_{n} n \operatorname{grad}\left(k_{B} T_{n}\right)+\mu_{n} k_{B} T_{n} \operatorname{grad} n .
$$

Now, observing that in [8] the diffusion coefficient is related to the carrier temperature by $D_{n c}^{\text {et }}=$ $\mu_{n c}^{\text {et }} k_{B} T_{n}^{\text {et }} / q$, one may recast (78) as

$$
\begin{aligned}
\mathbf{J}_{n}^{\mathrm{et}}= & q \mu_{n}^{\mathrm{et}} n \mathbf{F}+q \operatorname{grad}\left(n D_{n c}^{\mathrm{et}}\right) \\
& +q v_{n} \operatorname{grad}\left(\tilde{\tau} n v_{n}\right)+\mathbf{J}_{n h}^{\mathrm{et}}
\end{aligned}
$$

Comparing with Eq. (4) of [8], one sees that the last two terms of (80) are missing there. In particular, neglecting $\mathbf{J}_{n h}^{\text {et }}$ is equivalent to considering $\tau$ independent of position (compare with (69), (70)), hence to ignore impurity scattering. As for term $q v_{n} \operatorname{grad}\left(\tilde{\tau} n v_{n}\right)$, it originates from the contribution of the drift velocity inherent in the definition (69) of $\mathscr{B}_{n}$ (the latter, in turn, is fully equivalent to Eq. (6) of [8]). Hence the carrier transport, as modeled in [8], either spuriously incorporates in the diffusion coefficient a contribution from the drift velocity or (depending on which expression for the diffusion coefficient is actually used) neglects term $q v_{n} \operatorname{grad}\left(\tilde{\tau} n v_{n}\right)$ on the right hand side of the transport equation.

The treatment of the $M=3$ case is similar. It is worth noting that the necessity of a closure condition is apparently missing from the energy-transport model. A closer look shows that it is actually present in the definition of the energy-diffusion coefficient $B_{n}^{E}$, which plays in the equation for the energy-flux density the same role as $B_{n}$ in (72). In fact, the energy-diffusion coefficient embeds a moment of rank 4 in the same manner as the mobility defined by (74) embeds a moment of rank 2 . As a consequence, this brings about the drawback that the closure condition of the ET model is affected by the macroscopic model chosen for $B_{n}^{E}$.

\section{FURTHER DISCUSSION ON THE HD MODEL}

The starting point for the discussion is the expression of the energy flux $\mathbf{P}_{n}$. It is worth a reminder that the solution scheme replaces $n \mathbf{P}_{n}$ (taken from (53) or from its simplified form (55)) into the left hand side of (50). So doing, the rank-3 unknown $\mathbf{P}_{n}$ is eliminated and the equation is solved for the rank-2 unknown $T_{n}$. After that, the right hand side of (53) or (55) is known whence $P_{n}$ is determined. Finally, from the definition (49) of $\mathbf{P}_{n}$ one may recover its rank-3 portion $(m / 2) \overline{c_{n}^{2} \mathbf{c}_{n}}$ (the above corresponds to the more familiar procedure of solving the first of (45) for $n$, determining $\mathbf{J}_{n}$ from the second, and recovering $\mathbf{v}_{n}$ from $\mathbf{J}_{n}=-q n \mathbf{v}_{n}$ ). Using 
the rank-3 portion of $\mathbf{P}_{n}$ multiplied by $n$,

$$
\mathbf{Q}_{n}=n \frac{m}{2} \overline{c_{n}^{2} \mathbf{c}_{n}}
$$

and combining (49) and (55), one finds

$$
\begin{aligned}
\mathbf{Q}_{n} \simeq & -\frac{5}{2} k_{B} T_{n} n \mathbf{v}_{n} \\
& -\frac{\tau_{q n}}{m} \operatorname{grad}\left[n\left(\frac{1}{2} m v_{n}^{2} k_{B} T_{n}+\frac{5}{2}\left(k_{B} T_{n}^{\mathrm{eq}}\right)^{2}\right)\right] \\
& -\frac{\tau_{q n}}{m} n \frac{5}{2} k_{B} T_{n} q \mathbf{F}
\end{aligned}
$$

(consistently with section 4 , (3/2) $k_{B} T_{n} \gg(1 / 2) m v_{n}^{2}$ has been assumed in (49)). It is interesting to compare the above expression with others, taken from the literature. To this purpose, (82) is first rearranged following a few steps listed below; in the procedure, the first three definitions in (33) and the expression (45) of the current density are used.

1. The first and third terms on the right hand side of (82) are combined to yield

$$
\begin{aligned}
\left(\frac{\tau_{q n}}{\tau_{p n}}-1\right) \frac{5}{2} k_{B} T_{n} n \mathbf{v}_{n} \\
\quad+\frac{\tau_{q n}}{\tau_{p n}} \frac{\mu_{n}}{q} \frac{5}{2} k_{B} T_{n} \operatorname{grad}\left(n k_{B} T_{n}\right)
\end{aligned}
$$

2. In turn, the second term yields

$$
\begin{aligned}
-\frac{\tau_{q n}}{\tau_{p n}} \frac{\mu_{n}}{q}[ & \frac{1}{2} m v_{n}^{2} \operatorname{grad}\left(n k_{B} T_{n}\right) \\
& +\frac{1}{2} n k_{B} T_{n} \operatorname{grad}\left(m v_{n}^{2}\right) \\
& \left.+\frac{5}{2}\left(k_{B} T_{n}^{\mathrm{eq}}\right)^{2} \operatorname{grad} n\right] .
\end{aligned}
$$

3. Eqs. (83) and (84) are then added up. The first term in (84) can be neglected with respect to the last in (83) because the thermal part of the average kinetic energy is dominant. The second term in (84), which equals $-\tau_{q n} k_{B} T_{n} n v_{n} \operatorname{grad} v_{n}$, is neglected because it is proportional to the convective part of the current density (compare with (23)). The remain- ing terms yield

$$
\begin{aligned}
\mathbf{Q}_{n}= & \frac{5}{2}\left(\frac{\tau_{q n}}{\tau_{p n}}-1\right) k_{B} T_{n} n \mathbf{v}_{n}+ \\
+ & \frac{5}{2} \frac{\tau_{q n}}{\tau_{p n}} \frac{\mu_{n}}{q} k_{B}^{2} \\
& \times\left[T_{n} n \operatorname{grad} T_{n}+\left(T_{n}^{2}-\left(T_{n}^{\mathrm{eq}}\right)^{2}\right) \operatorname{grad} n\right] .
\end{aligned}
$$

This expression for $\mathbf{Q}_{n}$ is more convenient because it does not contain $v_{n}^{2}$, and is therefore taken as a starting point for the comparisons. The first issue is that of showing that the limiting case of (85) is consistent with the results of irreversible thermodynamics. This consistency must be sought since, as demonstrated in [17], the extension of the isothermal device equations is possible in order to incorporate heat conduction and generalized heat sources and sinks. To this purpose, letting

$$
\sigma_{n}=q \mu_{n} n, \quad \mathbf{U}_{n}=\frac{2 / 5}{\left(\tau_{q n} / \tau_{p n}\right)-1} \mathbf{Q}_{n},
$$

and using again the expression (45) of the current density, gives for (85)

$$
\begin{aligned}
\mathbf{U}_{n}= & -\sigma_{n} \frac{k_{B} T_{n}}{q} \mathbf{F}+\frac{k_{B}^{2}}{q^{2}} \frac{\sigma_{n} T_{n}}{\left(\tau_{q n} / \tau_{p n}\right)-1} \operatorname{grad} T_{n} \\
& +\sigma_{n} \frac{k_{B}^{2}}{q^{2}} \frac{\tau_{p n} T_{n}^{2}-\tau_{q n}\left(T_{n}^{\mathrm{eq}}\right)^{2}}{\tau_{q n}-\tau_{p n}} \operatorname{grad} \log n . \quad
\end{aligned}
$$

In the above, $\sigma_{n}$ is the electrical conductivity of the electrons, while $\mathbf{U}_{n}$ and $\mathbf{Q}_{n}$ are referred to as thermal current and heat flux, respectively. By taking now the limiting case $n=$ const and letting

$$
\begin{gathered}
L_{E E}=\sigma_{n}, \quad L_{E} T=\sigma_{n} \frac{k_{B}}{q}, \\
L_{T E}=-\sigma_{n} \frac{k_{B} T_{n}}{q}, \quad L_{T T}=\frac{k_{B}^{2}}{q^{2}} \frac{\sigma_{n} T_{n}}{\left(\tau_{q n} / \tau_{p n}\right)-1},
\end{gathered}
$$

(45) and (88) take the more compact form

$$
\begin{gathered}
\mathbf{J}_{n}=L_{E E} \mathbf{F}+L_{E T} \operatorname{grad} T_{n}, \\
\mathbf{U}_{n}=L_{T E} \mathbf{F}+L_{T T} \operatorname{grad} T_{n} .
\end{gathered}
$$

The coefficients (88) fulfill the Kelvin-Onsager rela- 
tion $L_{T E}=-T_{n} L_{E T}$ (see, e.g., [18, Sec. 7.5]). The thermal conductivity of the electrons, in turn, is given by

$$
\kappa_{n}=\frac{L_{T E} L_{E T}}{L_{E E}}-L_{T T}=\frac{k_{B}^{2}}{q^{2}} \frac{\sigma_{n} T_{n}}{\left(\tau_{p n} / \tau_{q n}\right)-1} .
$$

Letting

$$
\frac{1}{\left(\tau_{p n} / \tau_{q n}\right)-1}=\frac{5}{2}+g_{n}, \quad g_{n}=\frac{7 \tau_{q n}-5 \tau_{p n}}{2\left(\tau_{p n}-\tau_{q n}\right)},
$$

(90) provides the usual expression of the Wiedmann-Franz law. Finally, replacing (85) back into (49) one expresses the energy-flux density $n \mathbf{P}_{n}$ as

$$
\begin{aligned}
n \mathbf{P}_{n}= & \frac{5}{2} \frac{\tau_{q n}}{\tau_{p n}} k_{B} T_{n} n \mathbf{v}_{n}+\frac{5}{2} \frac{\tau_{q n}}{\tau_{p n}} \frac{k_{B}^{2}}{q^{2}} \sigma_{n} \\
& \times\left[T_{n} \operatorname{grad} T_{n}+\left(T_{n}^{2}-\left(T_{n}^{\mathrm{eq}}\right)^{2}\right) \operatorname{grad} \log n\right] .
\end{aligned}
$$

The above expression can be compared with the corresponding one in [12] (Eq. (17)). This is readily done if one neglects the convective part of the average kinetic energy in the definition of temperature there (Eq. (9)), and assumes a parabolic band. One then sees that both expressions of the energyflux density are proportional to the factor $\tau_{q n} / \tau_{p n}$, and that the convective part of it (i.e., that proportional to $n \mathbf{v}_{n}$ in (92)) is identical in the two cases. The other parts of the models (compare with Eqs. (13)-(15) of [12]) are also the same if recombination is neglected. In the traditional hydrodynamic model, the heat flux is expressed by means of Fourier's law $\mathbf{Q}_{n}=-\kappa_{n}^{\prime} \operatorname{grad} T_{n}$, where $\kappa_{n}^{\prime}$ is such that $\kappa_{n}^{\prime} /\left(\sigma_{n} T_{n}\right)$ $=$ const. This expression of $\mathbf{Q}_{n}$ is used for determining the last term at the right hand side of (49) and, in this respect, is viewed as the closure condition of the model. However, a number of difficulties can easily be envisaged in such a scheme. First, replacing Fourier's law directly into (49) implies letting $\tau_{q n}=\tau_{p n}$ in the convective part of $n \mathbf{P}_{n}$. Second, taking $\kappa_{n}^{\prime} /\left(\sigma_{n} T_{n}\right)=$ const is in contrast with (90) where, at constant $n, \tau_{p n} / \tau_{q n}$ may still depend on $T_{n}$ hence on position. Further difficulties arise from the mathematical viewpoint, since a slight change in $g_{n}$ induces large relative variations around zero in the coefficient of the highest-order operator in (50).
This may in fact be the case because $g_{n}$ ranges around -2 [5]. This problem does not occur if one uses the model proposed here and replaces $n \mathbf{P}_{n}$ taken from (55) or (92) into the left hand side of (50). Moreover, a fitting parameter such as $g_{n}$ of the standard hydrodynamic model is not needed anymore, since all coefficients are expressed in terms of the relaxation times.

A final comment is now necessary about the nonconvective part of $n \mathbf{P}_{n}$ in (92), which turns out to be different from that of [12]. Specifically, the substitution

$$
\begin{aligned}
-T_{n} \operatorname{grad} T_{n} \Leftrightarrow & T_{n} \operatorname{grad} T_{n} \\
& +\left(T_{n}^{2}-\left(T_{n}^{\mathrm{eq}}\right)^{2}\right) \operatorname{grad} \log n
\end{aligned}
$$

is necessary in order transform one equation into the other. This is easily traced back to the different closure condition of [12] which, using the current symbols and neglecting as usual the convective part of the average kinetic energy, reads

$$
\overline{\mathbf{c}_{n} \mathbf{c}_{n} \mathbf{c}_{n} \mathbf{c}_{n}}=\frac{5}{3}\left(\overline{\mathbf{c}_{n} \mathbf{c}_{n}}\right)^{2} \text {. }
$$

Using (94) gives rise to cancellation of a few terms in the derivation of $n \mathbf{P}_{n}$, which does not occur when using (31). In the model presented here the heat flux depends on $\operatorname{grad} T_{n}, \operatorname{grad} n$ and F. Among other things, such dependence may qualitatively explain the shift between the positions where $\mathbf{Q}_{n}=0$ and $\operatorname{grad} T_{n}=0$ occur, found by a full solution of the BTE [10]. One may also notice that in realistic cases the signs of $\operatorname{grad} T_{n}$ and $\operatorname{grad} n$ are opposite. As a consequence, the two terms may partially compensate each other in case of rapid spatial variations in the unknowns. Of course, extensive experimenting is still necessary in order to investigate the features of the model and complete the discussion about the closure condition.

In a recent paper, the derivation of the hydrodynamic model has been reworked from the thermodynamic viewpoint, using the entropy principle [19]. Among the outcomes of that theory is a constitutive relation for $\mathbf{Q}_{n}$ (Eq. (33)) whose convective part is identical to that of (85). The non-convective part, in turn, is made identical to that of (85) using the substitution (93). This hints that the closure conditions of [12] and [19] yield the same expressions for $n \mathbf{P}_{n}$ and $\mathbf{Q}_{n}$. This is indeed true as proven by direct calculation ${ }^{2}$. A final observation is that, since the

\footnotetext{
${ }^{2} \mathrm{R}$. Thoma, personal communication.
} 
heat flux in the uniform case can be calculated in a relatively easy way by a direct solution of the BTE, its expression $\mathbf{Q}_{n}=-(5 / 2)\left(1-\tau_{q n} / \tau_{p n}\right) k_{B} T_{n} n \mathbf{v}_{n}$ constitutes an alternative means for estimating $\tau_{q n}(\mathbf{F})$.

\section{CONCLUSIONS}

The structure of the moment equations has been analyzed for the purpose of deriving a general solution scheme. Each pair of two consecutive first-order PDEs is transformed into a second-order PDE (Eq. (26)) labeled $(M, M+1), M$ even, whose unknown is a rank $M$ tensor. Each pair is linked to the next one through a tensor of rank $M+2$, hence in any finite set of such pairs the number of unknowns exceeds the number of equations. A closer insight shows that the link is eliminated by neglecting the divergence of a rank $M+2$ tensor, this leading to the closure condition (31). The latter, supplemented with additional information (such as the diagonal nature of the tensor involved), renders a closed set of equations. As interesting feature of the closure condition is that it is intrinsic to the equations; in other terms, it can be stated for any set of consecutive pairs in a formal way. Another observation is that the grouping of the equations into pairs automatically pinpoints the closure condition at an even-rank tensor. This is consistent with the suggestion of closing at the fourth rank instead of using Fourier's law, that has recently been proposed in [11]. However, a comparison with the results of [11] is beyond the extent of this paper.

As an example of application, the first two pairs $(0,1),(2,3)$ have been examined after simplifying their tensor nature by assuming scalar relaxation times and taking the trace. It is worth noting that, by this procedure, an even-rank equation like (26) gives rise to an equation whose unknown is a scalar. This is particularly useful in view of the application of standard discretization techniques such as the Box Integration Method (see, e.g., [2]). The closure condition (31) yields the standard drift-diffusion model for $M=0$, and a more complete form of the hydrodynamic model for $M=2$.

Next, the form of the coefficients of (26) has briefly been examined and compared with that of the energy-transport model. It has been shown that the coefficients in the two cases are essentially different. Due to this, it has little meaning to carry out a comparison of the two models adopting the same mobility and/or diffusivity for both. This, in fact, would mask the differences inherent in the definition of the latter.

Finally, the more complete form of the hydrodynamic model proposed here has been compared with those of [12] and [19]. It has been shown that the closure condition here gives rise to a different form of the non-convective part of the heat flux (85) and, consequently, of the energy-flux density (92). This brings about the necessity of numerical experiments in order to assess the importance of each term in realistic situations. Formally, the presence of both grad $n$ and grad $T_{n}$ in (92) hints the possibility of a partial compensation in case of rapid spatial variations in the unknowns. Other features of the model are in common with those of [12] and [19], and confirm the insufficiency of the Fourier law in this framework. In particular, they are the presence of a convective part of the heat flux and a factor $\tau_{q n} / \tau_{p n}$ in the expression of the energy-flux density. Within the approximation of the model, factor $\tau_{q n} / \tau_{p n}$ is a function of the moments and impurity concentrations, hence of position. Finally, all coefficients are expressed in terms of the relaxation times, without resorting to independent information such as the Wiedmann-Franz law.

\section{Acknowledgments}

The authors wish to thank the Reviewers for their constructive remarks.

\section{References}

[1] M. Rudan, A. Gnudi, and W. Quade. A Generalized Approach to the Hydrodynamic Model of Semiconductor Equations, volume Process and Device Modeling for Microelectronics (edited by G. Baccarani), chapter 2, pages 109-154. Elsevier, 1993.

[2] M. Rudan and F. Odeh. Multi-Dimensional Discretization Scheme for the Hydrodynamic Model of Semiconductor Devices. COMPEL, 5(3):149-183, 1986.

[3] M. Rudan, F. Odeh, and J. White. Numerical Solution of the Hydrodynamic Model for a One-Dimensional Semiconductor Device. COMPEL, 6(3):151-170, 1987.

[4] A. Forghieri, R. Guerrieri, P. Ciampolini, A. Gnudi, M. Rudan, and G. Baccarani. A new discretization strategy of the semiconductor equations comprising momentum and energy balance. IEEE Trans. on CAD of ICAS, CAD7(2):231-242, 1988.

[5] A. Gnudi, F. Odeh, and M. Rudan. Investigation of nonlocal transport phenomena in small semiconductor devices. European Trans. on Telecommunications and Related Technologies, 1(3):307-312 (77-82), 1990.

[6] W. Quade, M. Rudan, and E. Schöll. Hydrodynamic simulation of impact-ionization effects in $p-n$ junctions. IEEE Trans. on CAD of ICAS, CAD-10(10):1287-1294, 1991.

[7] P. Ciampolini, A. Pierantoni, A. Liuzzo, and G. Baccarani. $3 D$ Simulation of Silicon Devices: Physical Models and Numerical Algorithms, volume Process and Device Modeling 
for Microelectronics (edited by G. Baccarani), chapter 2, pages 53-107. Elsevier, 1993.

[8] D. Chen, E.C. Kan, U. Ravaioli, C. Shu, and R.W. Dutton. An improved energy transport model including non-parabolicity and non-Maxwellian distribution effects. IEEE Electron Device Letters, 13(1):26-28, January 1992.

[9] D. Chen, E. Sangiorgi, M.R. Pinto, E.C. Kan, U. Ravaili, and R. W. Dutton. Analysis of Spurious Velocity Overshoot in Hydrodynamic Simulations. In P. Lloyd, A.R. Neureuther, and D. Rose, editors, Proc. of the NUPAD IV Conference, pages 109-114, Seattle, 1992. IEEE.

[10] M.A. Stettler, M.A. Alam, and M. Lundstrom. A Critical Assessment of the Hydrodynamic Transport Model Using the Scattering Matrix Approach. In P. Lloyd, A.R. Neureuther, and D. Rose, editors, Proc. of the NUPAD IV Conference, pages 97-102, Seattle, 1992. IEEE.

[11] T-W. Tang, S. Ramaswamy, and J. Nam. An Improved Hydrodynamic Transport Model for Silicon. IEEE Trans. on Electron Devices, ED-40(8), August 1993.

[12] R. Thoma, A. Emunds, B. Meinerzhagen, H.-J. Peifer, and W.L. Engl. Hydrodynamic Equations for Semiconductors with Nonparabolic Band Structure. IEEE Trans. on Electron Devices, ED-38(6), June 1991.

[13] M.C. Vecchi and L.G. Reyna. Generalized Energy Transport Model for Semiconductor Device Simulation. RC 19077 (83226), IBM Res., 1993.

[14] D. Ventura, A. Gnudi, and G. Baccarani. Multidimensional Spherical Harmonics Expansion of Boltzmann Equations for Transport in Semiconductors. Applied Mathematics Letters, 5(3):85-90, 1992.

[15] C.L. Gardner, D.J. Rose, and J.W. Jerome. Numerical methods for the hydrodynamic device model: Subsonic flow. IEEE Trans. on CAD of ICAS, CAD-8:501, 1989.

[16] W. Quade, E. Schöll, and M. Rudan. Impact-ionization within the hydrodynamic approach to semiconductor transport. Solid-State Electronics, 26(10):1493-1505, 1993.

[17] G.K. Wachutka. Rigorous Thermodynamic Treatment of Heat Generation and Conduction in Semiconductor Device Modeling. IEEE Trans. on CAD of ICAS, CAD9(11):1141-1149, 1990.

[18] J.M. Ziman. Electron and Phonons. Clarendon Press, Oxford, 1960.

[19] A.M. Anile and S. Pennisi. Thermodynamic Derivation of the Hydrodynamical Model for Charge Transport in Semiconductors. Physical Review B, 46(20):13186-13193, November 1992-II.

\section{Biographies}

MASSIMO RUDAN was born in Bologna, Italy, in 1949. He received a degree in Electrical Engineering in 1973 and a degree in Physics in 1976, both from the University of Bologna, Bologna, Italy.

After serving as a Naval officer, he joined the Dipartimento di Elettronica, Informatica e Sistemistica (DEIS) of the University of Bologna in 1975, where he investigated the physical properties of the MOs structures and the problems of analytical modelling of semiconductor devices. From 1978 he has been teaching an annual course of Quantum Electronics in the Faculty of Engineering of the same University, firstly as Lecturer and then as Associate Professor. Since 1983 he has been working in a group involved in numerical analysis of semiconductor devices, acting as Task leader in a number of EEC-supported Projects in the area of CAD for VLSI.

In 1986 he has been a visiting scientist, on a one-year assignment, at the IBM Thomas J. Watson Research Center at Yorktown Heights, NY, studying the discretization techniques for the higher-order moments of the Boltzmann Transport Equation. He was again with IBM for shorter periods afterwards, investigating and implementing impact-ionization models based on the average energy of the carriers. In 1990 he was appointed Full Professor of Microelectronics at the University of Bologna.

GIORGIO BACCARANI was born in Modena, Italy, in 1943. He received a degree in Electrical Engineering in 1967, and in Physics in 1969, both from the University of Bologna, Bologna, Italy. In 1969 he joined the Bell Laboratories, Murray Hill, NJ, as a limited-term Member of the Technical Staff, working in the area of electron-device processing.

In 1970 he became a Research Assistant at the University of Bologna, where he investigated the physical properties of MOs structures and transport effects in semiconductor materials and devices. Since 1972 he has been teaching an annual course in the same Institution, first on Quantum Electronics and then on Digital Circuit Design. In 1980 he was appointed Full Professor of Digital Electronics at the University of Bologna.

In 1981 he was on a one-year assignment at the IBM Thomas J. Watson Research Center, Yorktown Heights, NY, where he investigated the feasibility of a $1 / 4$ micrometer MOs process from the standpoint of the physical limitations affecting the device performance. In 1983 and 1989 he was again a visiting scientist at IBM, investigating advanced physical models for device simulation, and silicon-based heterojunction bipolar transistors, respectively.

Since 1983 he has been heading a group involved in numerical analysis of semiconductor devices, acting as partner leader in the context of four successive EEC-supported Projects in the area of CAD for VLSI. His current research interests include numericaldevice simulation, synthesis of analog circuits, analog and digital architectures for image processing and integrated-circuits design. 

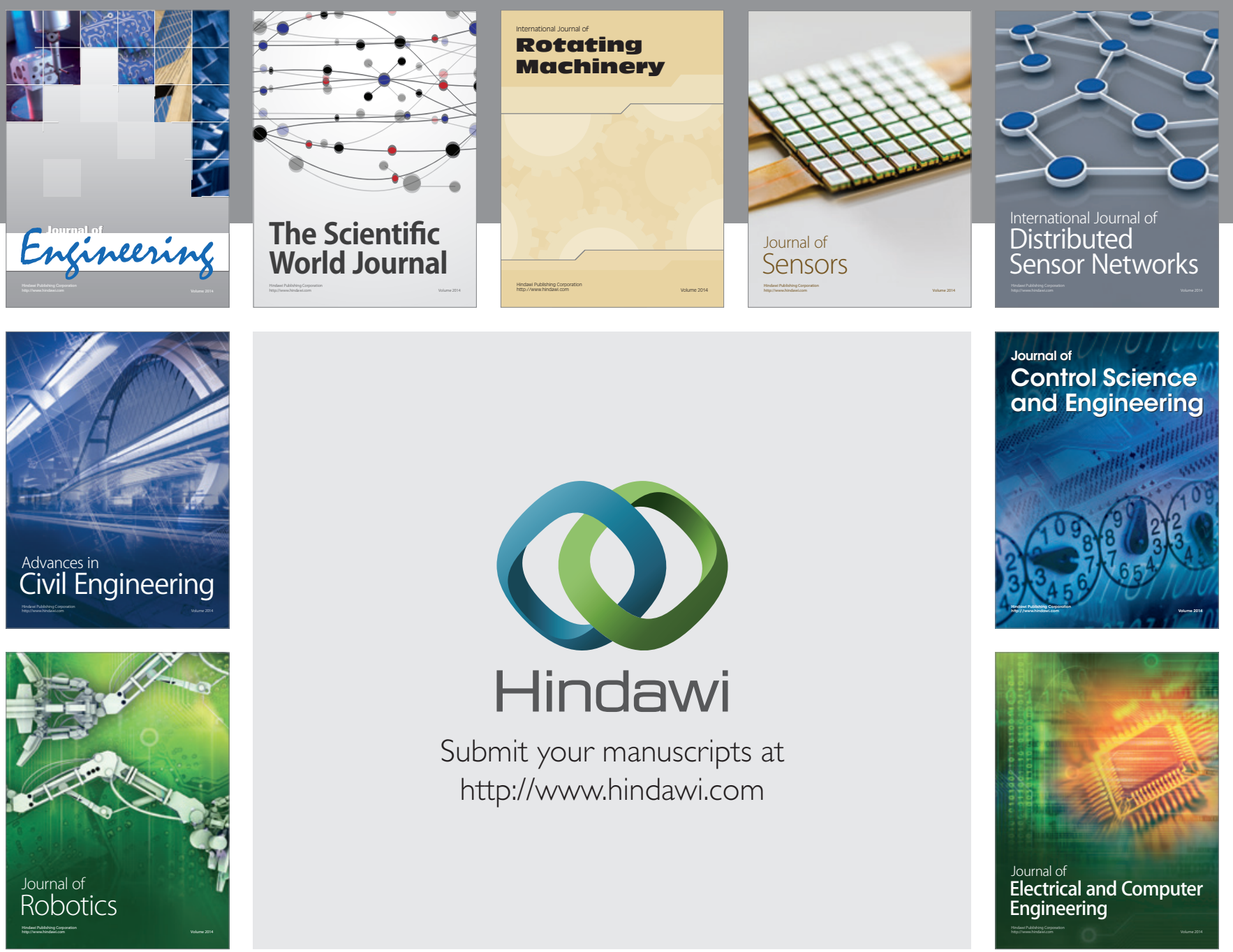

Submit your manuscripts at

http://www.hindawi.com
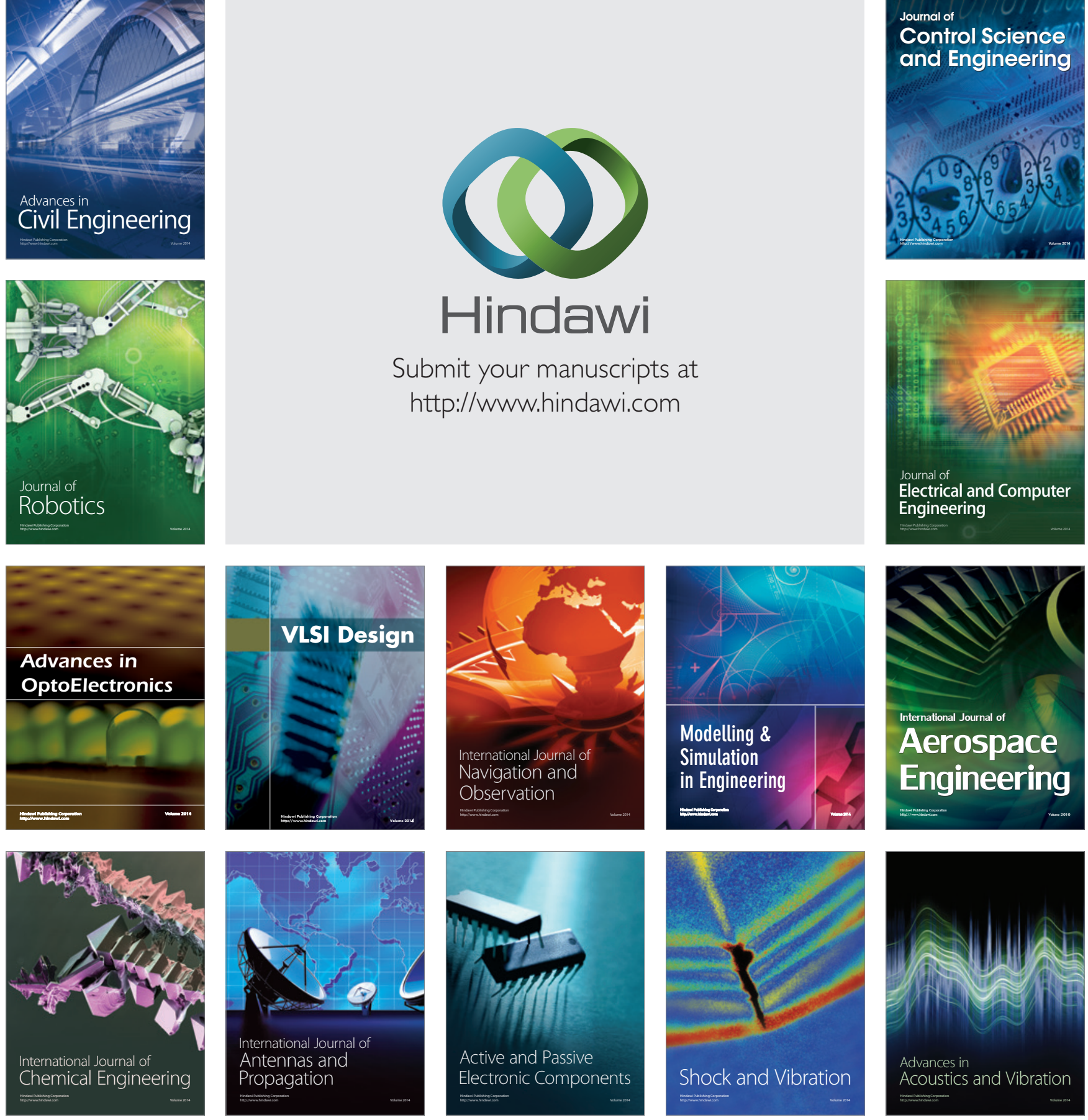